\title{
Lycium ruthenicum extract alleviates high-fat diet-induced nonalcoholic fatty liver disease via enhancing the AMPK signaling pathway
}

\author{
JIAYAO LIN, YU ZHANG, XINQING WANG and WENWEN WANG \\ Department of Geriatrics, Huashan Hospital, Fudan University, Shanghai 200040, P.R. China
}

Received August 13, 2014; Accepted April 30, 2015

DOI: $10.3892 / \mathrm{mmr} .2015 .3840$

\begin{abstract}
Nonalcoholic fatty liver disease (NAFLD) is a common liver disease that currently has no standard treatment. The present study aimed to evaluate the effects of Lycium ruthenicum extract (LRE) on high-fat diet (HFD)-induced NAFLD, and elucidate the underlying molecular mechanisms. To determine the hepatoprotective effect of LRE, C57BL/6 mice were fed a normal control diet, high-fat diet (HFD), HFD supplemented with $2 \mathrm{~g} / \mathrm{kg}$ LRE, or HFD supplemented with $5 \mathrm{~g} / \mathrm{kg}$ LRE. Treatment with LRE markedly decreased the levels of triglycerides, total cholesterol, aspartate aminotransferase and alanine aminotransferase in the serum of mice fed a HFD, and improved glucose metabolism and insulin sensitivity in NAFLD mice. In addition, treatment with LRE significantly decreased the expression levels of sterol regulatory element-binding protein $1 \mathrm{c}$ and fatty acid synthase, and markedly increased the expression of peroxisome proliferator-activated receptor $\alpha$ and peroxisome proliferator-activated receptor $\gamma$ co-activator $1 \alpha$. Furthermore, LRE treatment significantly increased the activation of adenosine monophosphate-activated protein kinase (AMPK) in the liver. These results suggested that LRE is able to suppress lipid accumulation in HFD-fed C57BL/6 mice via enhancement of the AMPK pathway.
\end{abstract}

\section{Introduction}

Non-alcoholic fatty liver disease (NAFLD) is one of the most common types of liver disease worldwide. The prevalence of NAFLD is $\leq 30 \%$ in Western countries and $~ 5-30 \%$ in Asia-Pacific nations (1). NAFLD is characterized by excessive hepatic lipid accumulation, hepatic insulin resistance, and hepatic inflammation, which lead to nonalcoholic

Correspondence to: Dr Yu Zhang, Department of Geriatrics, Huashan Hospital, Fudan University, 12 Middle Wulumuqi Road, Shanghai 200040, P.R. China

E-mail: yu_zhangsh@126.com

Key words: Lycium ruthenicum extract, nonalcoholic fatty liver disease, lipid accumulation, AMPK pathway steatohepatitis (NASH), and finally hepatic fibrosis or cirrhosis (2). Numerous strategies have been proposed to improve NAFLD, including diet control, weight control and exercise; however, in practice, it is difficult to achieve and even more difficult to maintain for the majority of patients. There is currently no Food and Drug Administration approved treatment for NAFLD; therefore, the development of efficacious and safe drugs for the treatment of NAFLD is required.

Recently, the application of natural herbs for the treatment of NAFLD has received wide attention, due to their safety and efficacy (3). It has previously been reported that treatment with water-extracted Lycium barbarum suppresses liver fibrosis via downregulation of liver inflammation in rats with carbon tetrachloride-induced liver injury (4). In addition, Lycium barbarum polysaccharide has been shown to exhibit a protective effect in NASH-induced hepatic injury (5). Another species of the Lycium genus, Lycium ruthenicum, is a traditional Tibetan medicine used for the treatment of heart disease, hypertension and climacteric syndrome (6). Numerous studies have reported that Lycium ruthenicum has various pharmacological roles, including anti-oxidation, immunoregulation and hypoglycemic activity (7-9). However, the effects of Lycium ruthenicum on fat metabolism in high-fat diet (HFD)-induced NAFLD remain unclear. The present study aimed to explore the effects of Lycium ruthenicum in the treatment of HFD-induced NAFLD, and to determine its underlying mechanisms.

\section{Materials and methods}

Preparation of Lycium ruthenicum extract (LRE). The raw Lycium ruthenicum was obtained from Chaiqi Agricultural Development Co., Ltd. (Qinghai, China). The Lycium ruthenicum fruit $(500 \mathrm{~g})$ was immersed in $51 \mathrm{ddH}_{2} \mathrm{O}$ for $2 \mathrm{~h}$, and decocted twice at boiling temperature for $2 \mathrm{~h}$. The decocted liquid was then collected, filtrated with 100 mesh fabric, concentrated under reduced pressure, and stored at $4^{\circ} \mathrm{C}$ until further use.

Experimental animals. Six-week-old male C57BL/6J mice $(n=80)$ were purchased from the Animal Center of Huashan Hospital, Fudan University (Shanghai, China). The mice were maintained at a constant temperature of $22 \pm 1^{\circ} \mathrm{C}$ and relative humidity of $55 \pm 5 \%$, and underwent a $12 \mathrm{~h}$ light-dark cycle. 
The mice had access to food and water ad libitum. After 1 week acclimatization, the mice were randomly divided into four groups ( $\mathrm{n}=10 /$ group): Normal control diet (NCD; 100\% standard chow), HFD, HFD supplemented with low-dose LRE ( $2 \mathrm{~g} / \mathrm{kg} / \mathrm{day})$, and HFD supplemented with high-dose LRE $(5 \mathrm{~g} / \mathrm{kg} / \mathrm{day})$. The HFD consisted of $73 \%$ standard chow, $20 \%$ lard, $4 \%$ sucrose, $2 \%$ milk and $1 \%$ cholesterol. After 12 weeks of LRE treatment, the mice were anesthetized with pentobarbital (40 mg/kg body weight; Sigma-Aldrich, St. Louis, MO, USA) in order for liver biopsy to be conducted. Body weight and food intake for each mouse were recorded weekly throughout the study. At the end of the experiment, the mice were fasted overnight and sacrificed by $\mathrm{CO}_{2}$ asphyxiation. Blood and liver samples were harvested for further analyses. All animal experiments were approved by the Animal Care and Use Committee of Huashan Hospital, Fudan University (Shanghai, China).

Serum biochemical analysis. The blood samples were obtained by cardiac puncture, and centrifuged at $1,000 \mathrm{x}$ g for $10 \mathrm{~min}$ at $4^{\circ} \mathrm{C}$, in order to obtain serum samples. The levels of triglycerides (TG), total cholesterol (TC), aspartate aminotransferase (AST) and alanine aminotransferase (ALT) were determined using an automatic blood chemistry analyzer (Dri-Chem 4000i; Fujifilm, Tokyo, Japan).

Glucose tolerance test (GTT) and insulin tolerance test (ITT). Measurements of intraperitoneal (ip)GTT and ITT were performed as previously described (10). At the end of 12 weeks treatment, all of the mice were subjected to an ipGTT, following an overnight fast (12 h). An intraperitoneal glucose bolus ( $2 \mathrm{~g} / \mathrm{kg}$ body weight; Sigma-Aldrich) was administered to the conscious, unrestrained mice. The blood glucose levels were determined from tail blood samples taken at 0 (prior to glucose administration), 30, 60, 90, 120 and $150 \mathrm{~min}$ after the glucose injection, using the Freestyle Blood Monitoring system (Abbott Diabetes Care, Inc., Alameda, CA, USA). For ITT, the mice were subjected to a $6 \mathrm{~h}$ fast, followed by an i.p. injection of insulin (1 U/kg body weight; Sigma-Aldrich). Blood glucose concentrations were measured in the same manner as for ipGTT.

Western blot analysis. The liver tissue was washed with ice-cold phosphate-buffered saline and lysed with Radioimmunoprecipitation Cell Lysis Buffer (Gibco Life Technologies, Carlsbad, CA, USA) for protein extraction. The cell lysates were collected by centrifugation at $13,000 \mathrm{x} \mathrm{g}$ for $10 \mathrm{~min}$ at $4^{\circ} \mathrm{C}$, and protein concentrations were determined using the Bio-Rad Protein Assay kit (Bio-Rad Laboratories, Inc., Hercules, CA, USA). The protein samples (30 $\mu \mathrm{g}$ protein/lane) were separated by $10 \%$ SDS-PAGE and transferred onto polyvinylidene fluoride membranes (EMD Millipore, Billerica, MA, USA). The membranes were blocked with Tris-buffered saline $(50 \mathrm{mmol} / \mathrm{l} \mathrm{NaCl}$ and $10 \mathrm{mmol} / \mathrm{l} \mathrm{Tris}$; $\mathrm{pH}$ 7.4) containing 5\% non-fat milk, and were then incubated with the following rabbit monoclonal primary antibodies (dilution 1:500): Anti-peroxisome proliferator-activated receptor (PPAR)- $\alpha$ (sc-13040), anti-peroxisome proliferator-activated receptor $\gamma$ co-activator (PGC)-1 $\alpha$ (sc-291222), anti-sterol regulatory element-binding protein (SREBP)-1c (sc-366), anti-fatty acid synthase (FAS; sc-1023) and anti-phosphorylated (p)-adenosine monophosphate-activated protein kinase (AMPK; sc-101631) (Santa Cruz Biotechnology, Inc., Dallas, TX, USA), and anti- $\beta$-actin (1:1,000 dilution; cat. no. AM4302; Invitrogen Life Technologies, Carlsbad, CA, USA) at $4^{\circ} \mathrm{C}$ overnight. The blots were subsequently washed three times with tris-buffered saline containing $0.05 \%$ Tween-20, and incubated with horseradish peroxidase-conjugated secondary antibody (1:3,000; cat. no. sc-2370; Santa Cruz Biotechnology, Inc.) at room temperature for $1 \mathrm{~h}$. Protein-antibody complexes were visualized using the Enhanced Chemiluminescence Western Blotting Detection system (GE Healthcare, Vélizy-Villacoublay, France), according to the manufacturer's instructions. $\beta$-actin was used as an internal control. Protein level quantification was conducted using ImageJ version 1.47 (National Institutes of Health, Bethesda, MA, USA).

Statistical analysis. All of the data are expressed as the mean \pm standard deviation. Statistical comparisons between the experimental groups were made using one-way analysis of variance and Duncan's multiple-comparison test, using SPSS 13.0 software (SPSS Inc., Chicago, IL, USA). P<0.05 was considered to indicate a statistically significant difference.

\section{Results}

Effects of LRE on food consumption and body weight in HFD-fed mice. C57BL/6 mice were fed an NCD or HFD for 12 weeks, with or without LRE treatment. Food intake and body weight were measured weekly. As shown in Fig. 1A, there was no significant difference between the four groups, with regards to food consumption. After 12 weeks, the average body weight of the HFD mice was significantly increased to $47.92 \mathrm{~g}$, from an initial weight of $21.43 \mathrm{~g}$, whereas the weight of the mice in the NCD group increased to only $32.16 \mathrm{~g}$. The average body weight of the mice treated with low-LRE or high-LRE was lower, as compared with the mice in the HFD group, and the final average body weights were 42.31 and $38.24 \mathrm{~g}$, respectively (Fig. 1B).

Effects of LRE on HFD-induced serum levels of TG, TC, AST and ALT. To determine the effects of LRE on biochemical alterations, the serum levels of TC, TG, AST and ALT were measured. As shown in Fig. 2, after 12 weeks of the HFD, the serum levels of TC, TG, AST and ALT were significantly elevated in the HFD group, as compared with those of the NCD group, whereas treatment with LRE reversed this effect.

Treatment with LRE improves glucose metabolism and insulin resistance. Insulin resistance is regarded as a key factor in the pathogenesis of NAFLD (11). To evaluate the effects of LRE on glucose homeostasis and insulin sensitivity in HFD-fed mice, an ipGTT and ITT were performed after 12 weeks of LRE treatment. As shown in Fig. 3, fasting and postprandial hyperglycemia were evident in the HFD group, as compared with the NCD group at all time points of the glucose tolerance curve. The blood glucose levels in the HFD group reached a peak $30 \mathrm{~min}$ after i.p. glucose loading, and were marginally decreased by $150 \mathrm{~min}$. Conversely, treatment with LRE resulted in a significant decrease in blood glucose levels 
A

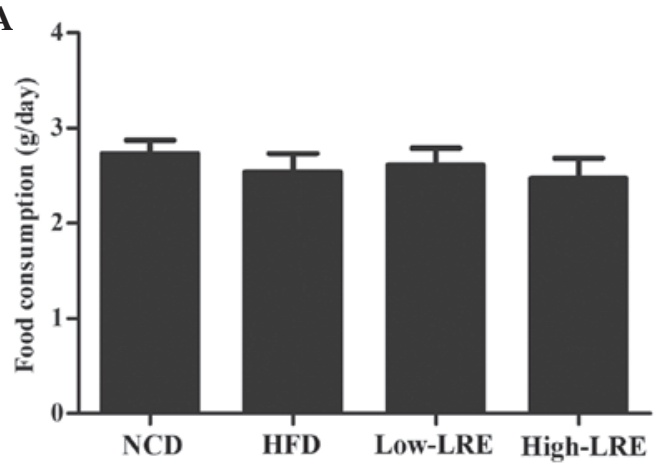

B

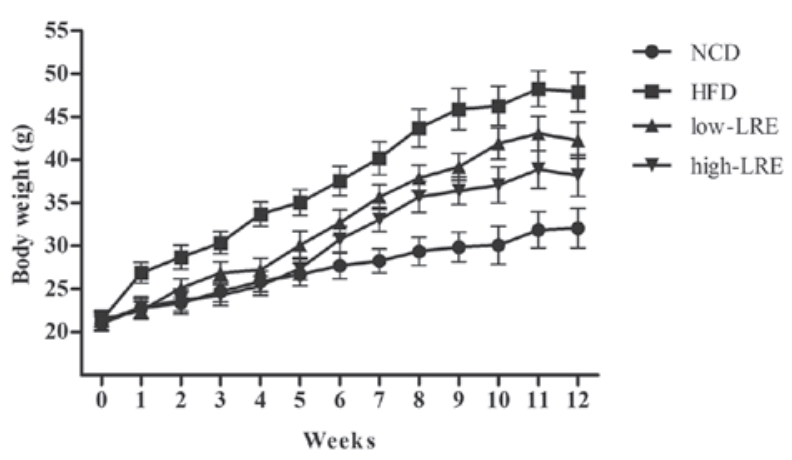

Figure 1. Effects of LRE treatment on food consumption and body weight in C57BL/6 mice fed a HFD. (A) Food consumption and (B) bodyweight of mice fed an NCD or HFD with or without LRE ( 2 and $5 \mathrm{mg} / \mathrm{kg}$ ). LRE was administered daily by oral gavage for 12 weeks. Data are presented as the mean \pm standard deviation (n=10/group). LRE, Lycium ruthenicum extract; NCD, normal control diet; HFD, high-fat diet.

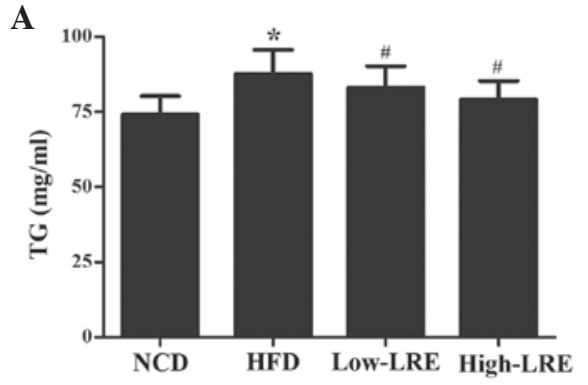

C

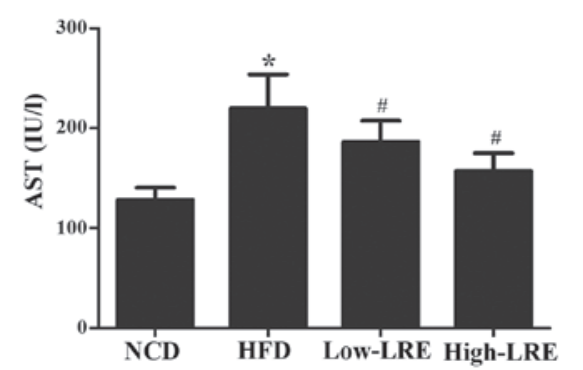

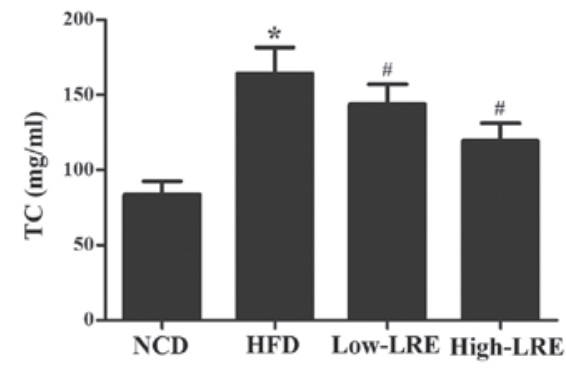

D

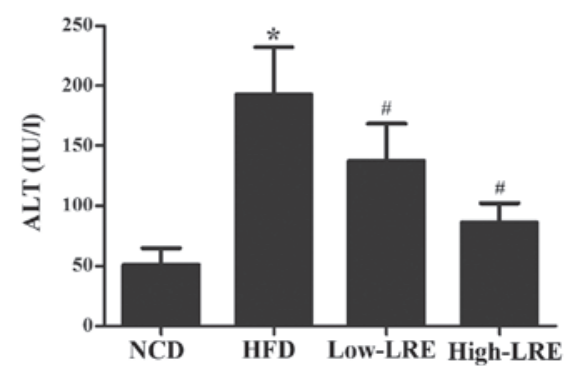

Figure 2. Effects of LRE treatment on serum TG, TC, AST and ALT levels in HFD-fed mice. Serum levels of (A) TG, (B) TC, (C) AST and (D) ALT in C57BL/6 mice fed an NCD or HFD with or without LRE ( 2 and $5 \mathrm{mg} / \mathrm{kg}$ ). LRE was administered daily by oral gavage for 12 weeks. Data are expressed as the mean \pm standard deviation. " $\mathrm{P}<0.05$, compared with the NCD group; ${ }^{*} \mathrm{P}<0.05$, compared with the HFD group. LRE, Lycium ruthenicum extract; NCD, normal control diet; HFD, high-fat diet; TG, triglycerides; TC, total cholesterol; AST, aspartate aminotransferase; ALT, alanine aminotransferase.

A

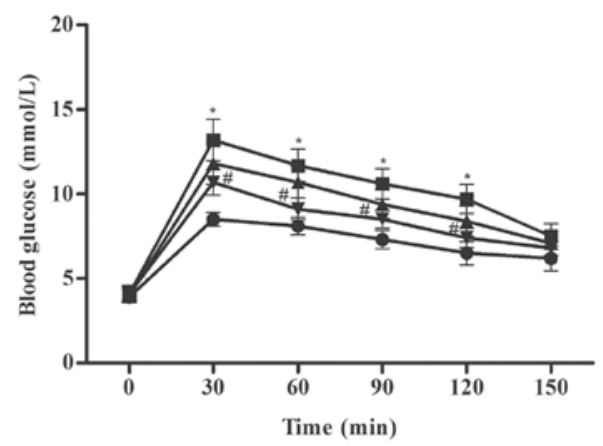

B

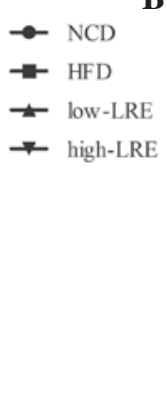

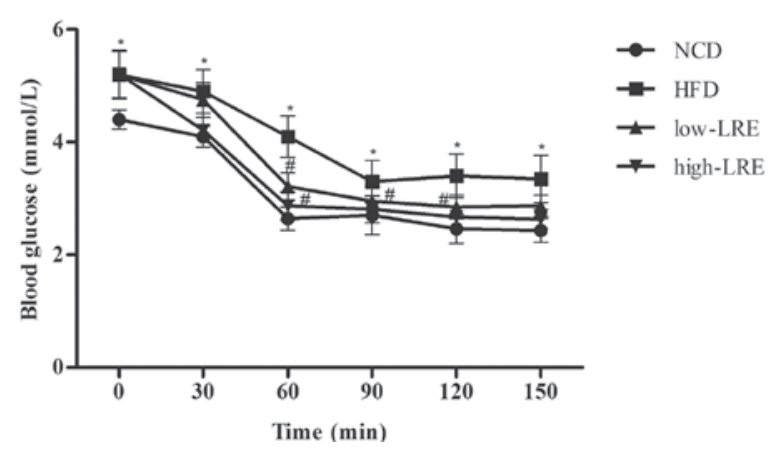

Figure 3. Effects of LRE treatment on glucose tolerance and insulin resistance. (A) Blood glucose levels of each mouse were recorded 0, 30, 60, 90, 120 and 150 min after an intraperitoneal injection of D-glucose after a 12-week induction of NAFLD. (B) Blood glucose levels were assessed after an intraperitoneal injection with recombinant insulin, at 0,30,60,90, 120 and 150 min after a 12-week induction of NAFLD. Data are expressed as the mean \pm standard deviation. ${ }^{*} \mathrm{P}<0.05$, compared with the NCD group; ${ }^{\prime} \mathrm{P}<0.05$, compared with the HFD group. NAFLD, non-alcoholic fatty liver disease; LRE, Lycium ruthenicum extract; NCD, normal control diet; HFD, high-fat diet. 

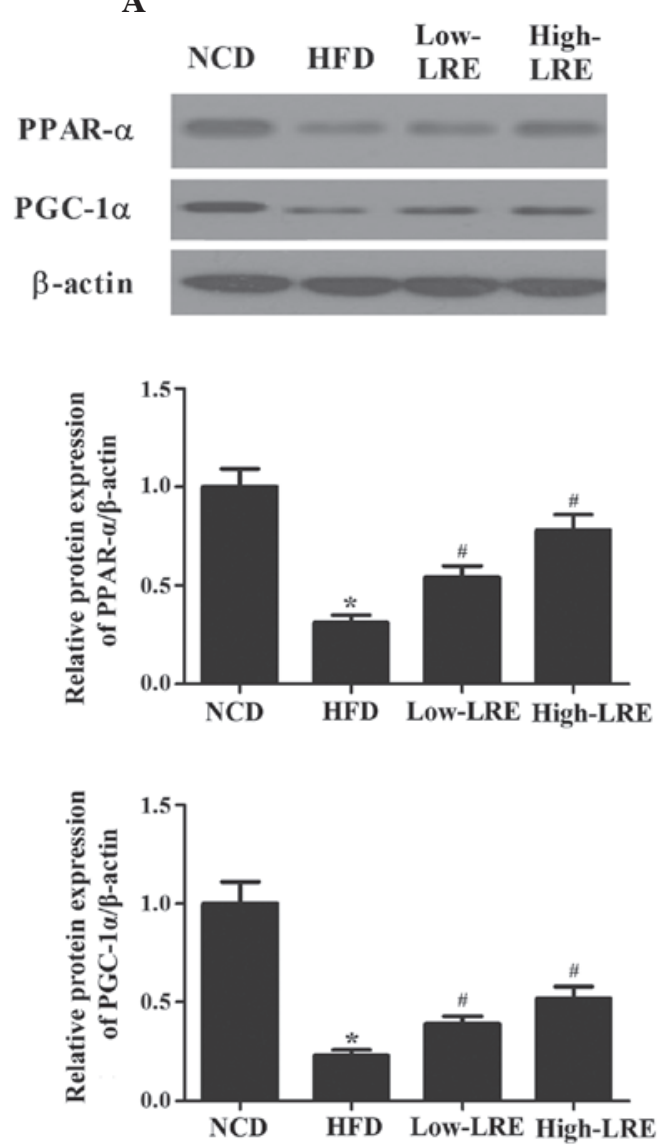

B
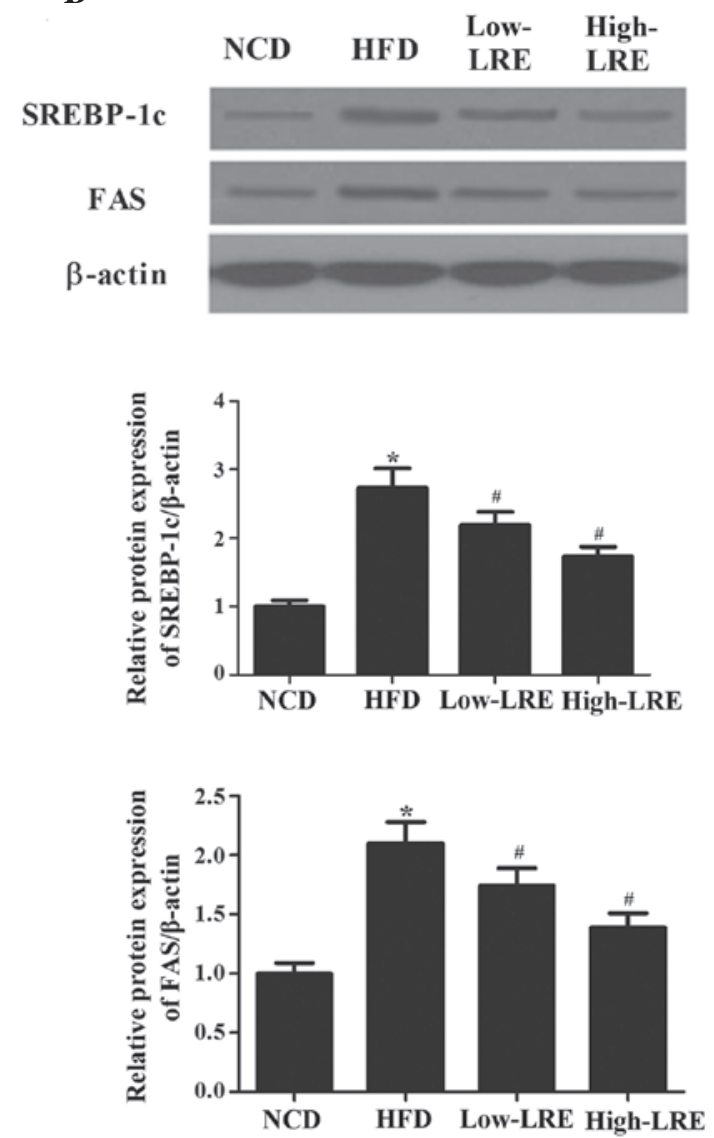

Figure 4. Effects of LRE treatment on lipid metabolism-associated hepatic protein expression in HFD-fed mice. C57BL/6 mice were fed an NCD or HFD with or without LRE ( 2 and $5 \mathrm{mg} / \mathrm{kg}$ ). LRE was administered daily by oral gavage for 12 weeks. Protein expression levels of hepatic (A) PPAR- $\alpha$ and PGC-1 $\alpha$, and (B) SREBP-1c and FAS were detected by western blotting, and representative images are shown. All experiments were repeated at least three times. Data are expressed as the mean \pm standard deviation. "P $<0.05$, compared with the NCD group; ${ }^{*} \mathrm{P}<0.05$, compared with the HFD group. LRE, Lycium ruthenicum extract; NCD, normal control diet; HFD, high-fat diet; PPAR- $\alpha$, peroxisome proliferator-activated receptor- $\alpha$; PGC-1 $\alpha$, peroxisome proliferator-activated receptor $\gamma$ co-activator-1 $\alpha$; SREBP-1C, sterol regulatory element-binding protein-1c; FAS, fatty acid synthase.
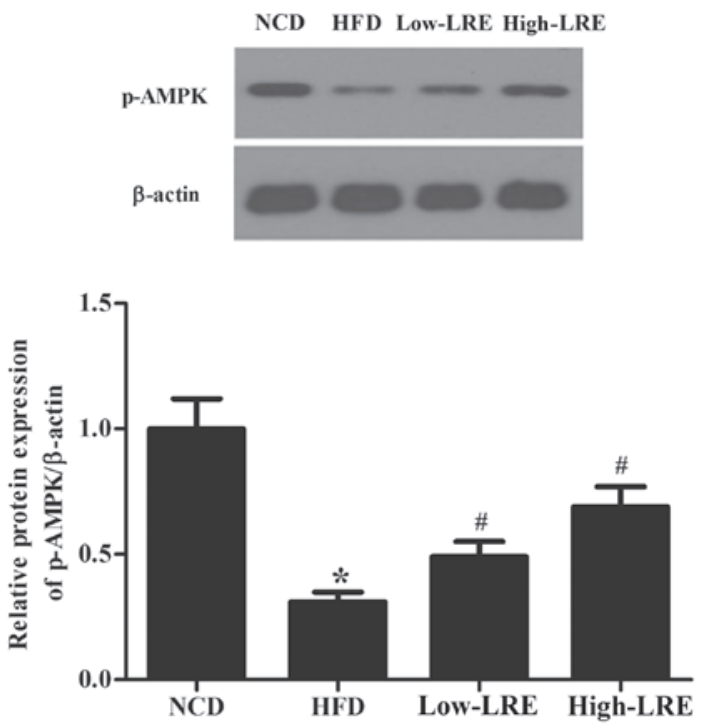

Figure 5. Effects of LRE treatment on the AMPK signaling pathway. LRE activated AMPK signaling in hepatic tissue. Total protein was collected from liver tissue. The protein expression levels were normalized to $\beta$-actin levels. All experiments were repeated at least three times. Data are expressed as the mean \pm standard deviation. " $\mathrm{P}<0.05$, compared with the NCD group; ${ }^{\#} \mathrm{P}<0.05$, compared with the HFD group. LRE, Lycium ruthenicum extract NCD, normal control diet; HFD, high-fat diet; AMPK, adenosine monophosphate-activated protein kinase. between 0 and 150 min of the GTT, thus suggesting that LRE was able to improve glucose tolerance (Fig. 3A). Furthermore, treatment with LRE significantly improved HFD-induced insulin resistance (Fig. 3B). These results suggest that treatment with LRE may improve glucose metabolism and insulin sensitivity in mice with HFD-induced NAFLD.

Effects of LRE on hepatic lipogenesis. Lipid metabolism has an important role in NAFLD (12). To investigate the effects of LRE on lipogenesis in the liver, numerous key transcriptional regulators of lipid metabolism were examined by western blotting: PPAR- $\alpha$, PGC-1 $\alpha$, SREBP 1c and FAS. As shown in Fig. 4, LRE treatment increased the protein expression levels of PPAR- $\alpha$ and PGC-1 $\alpha$ (Fig. 4A), and decreased the expression levels of SREBP-1c and FAS (Fig. 4B) $(\mathrm{P}<0.05)$, as compared with the HFD group. These results suggest that treatment with LRE is able to improve hepatic lipid metabolism through the suppression of lipogenesis and promotion of fat degradation.

LRE increases hepatic AMPK expression. AMPK has an important role in energy metabolism regulation in mammals (13). To determine the underlying mechanism of LRE on NAFLD, AMPK phosphorylation was examined by 
western blotting. As shown in Fig. 5, a HFD downregulated the expression of p-AMPK in the liver, whereas treatment with LRE markedly increased the expression levels of $\mathrm{p}$-AMPK.

\section{Discussion}

NAFLD is a metabolic syndrome characterized by abnormal hepatic lipid formation, without excessive alcohol intake. NAFLD is strongly associated with obesity and insulin resistance; therefore, a reciprocal cause-effect relationship has been suggested, which is associated with a vicious pathophysiological cycle (14). There are currently no specific or conventional treatments available for NAFLD; therefore, recent research has focused on the underlying molecular mechanisms of NAFLD development (15). The results of the present study demonstrated that LRE supplementation significantly decreased the serum levels of TG, TC, AST and ALT in HFD-fed mice. Treatment with LRE also attenuated hepatic lipid accumulation, by decreasing the expression levels of SREBP-1c and FAS, and increasing the expression levels of PPAR- $\alpha$ and PGC-1 $\alpha$. In addition, LRE treatment significantly increased hepatic activation of AMPK.

Serum TC, TG, AST and ALT are important clinical indicators of liver function. Insulin resistance is a major pathophysiological feature associated with NAFLD, which is hypothesized to contribute to the initiation and progression of NAFLD (16). NAFLD is characterized by reduced insulin sensitivity across the whole body, particularly in liver and adipose tissue. Insulin resistance results in excess accumulation of dietary fat, and enhanced delivery of free fatty acids to the liver. Increased levels of fatty acids impair fatty acid oxidation, and upregulate de novo lipogenesis (17). The present study showed that a HFD resulted in impaired glucose tolerance and insulin resistance; however, treatment with LRE was able to reverse the impaired glucose tolerance and insulin sensitivity. These results suggested that LRE may effectively prevent HFD-induced NAFLD, which is positively correlated with a reduction in the serum levels of TC, TG, AST, ALT and glucose.

It has previously been reported that SREBPs and PPAR- $\alpha$ are required for de novo lipogenesis and lipolysis in hepatic lipid metabolism (18). SREBPs regulate the expression of lipogenic enzymes, such as acetyl-CoA carboxylase (ACC), FAS and 3-hydroxy-3-methylglutarylcoenzyme A reductase (19). A previous study demonstrated that inhibition of SREBP-1c by gene knockout was able to decrease hepatic lipid accumulation (20). PPAR- $\alpha$ has an important role in fatty acid metabolism by upregulating the expression of numerous genes associated with mitochondrial fatty acid and peroxisome fatty acid oxidation, as well as numerous other aspects of cellular fatty acid metabolism (21). It has previously been shown that in NAFLD, hepatic PPAR- $\alpha$ levels are significantly decreased, and activating PPAR- $\alpha$ may prevent the development of NAFLD (22). PGC-1 $\alpha$ is a transcription co-activator, which is involved in mitochondrial biogenesis. PGC- $1 \alpha$ binds to PPAR $-\gamma$ and activates PPAR $-\gamma$ transactivity, which is associated with the regulation of carbohydrate and lipid metabolism (23). The present study demonstrated that treatment with LRE significantly decreased SREBP-1c and FAS expression levels, and markedly increased PPAR- $\alpha$ and PGC-1 $\alpha$ expression levels. These results indicated that LRE may dose-dependently prevent hepatic lipid accumulation by regulating lipid metabolism in HFD-fed mice.

NAFLD is associated with inhibition of AMPK and activation of SREBP-1 (24,25). Hepatic activation of AMPK suppresses anabolic processes, such as cholesterol and TG biosynthesis, by reducing the activities of SREBP-1 and FAS (26). Furthermore, AMPK activation promotes catabolic processes, including fatty acid $\beta$-oxidation by inactivation of ACC, and promotion of carnitine palmitoyltransferase-1 activity $(27,28)$. The present study demonstrated that phosphorylation of AMPK in HFD-fed mice was markedly decreased, and LRE treatment significantly recovered the phosphorylation of AMPK in liver tissue. These results suggested that LRE may suppress HFD-induced lipid accumulation through the AMPK signaling pathway.

In conclusion, treatment with LRE was able to enhance insulin sensitivity and suppress lipid accumulation in the liver of HFD-fed C57BL/6 mice, via enhancement of the AMPK signaling pathway. These results suggested that LRE may be a potential botanical target for the development of novel therapeutic alternatives for the treatment of NAFLD.

\section{Acknowledgements}

The present study was supported by funding from the Shanghai Medical Association Geriatrics Specialist Branch (grant no. LN201104).

\section{References}

1. Alba L and Lindor K: Review article: Non-alcoholic fatty liver disease. Aliment Pharmacol Ther 17: 977-986, 2003.

2. Jaskiewicz K, Rzepko R and Sledzinski Z: Fibrogenesis in fatty liver associated with obesity and diabetes mellitus type 2. Dig Dis Sci 53: 785-788, 2008.

3. Dong H, Lu FE and Zhao L: Chinese herbal medicine in the treatment of nonalcoholic fatty liver disease. Chin J Integr Med 18: 152-160, 2012.

4. Wu PS, Wu SJ, Tsai YH, Lin YH and Chao JC: Hot water extracted Lycium barbarum and Rehmannia glutinosa inhibit liver inflammation and fibrosis in rats. Am J Chin Med 39: 1173-1191, 2011.

5. Xiao J, Liong EC, Ching YP, Chang RC, Fung ML, Xu AM, So KF and Tipoe GL: Lycium barbarum polysaccharides protect rat liver from non-alcoholic steatohepatitis-induced injury. Nutr Diabetes 3: e81, 2013.

6. Ni W, Gao T, Wang H, Du Y,Li J,LiC, Wei Land Bi H: Anti-fatigue activity of polysaccharides from the fruits of four Tibetan plateau indigenous medicinal plants. J Ethnopharmacol 150: 529-535, 2013.

7. Li J, Li SZ, Feng WJ and Yuan H: In vitro antioxidant and free radical scavenging activities of total flavonoids from the leaves of Lycium ruthenicum Murr. Food Sci 13: 061, 2010.

8. Peng Q, Song J, LV X, Wang Z, Huang L and Du Y: Structural characterization of an arabinogalactan-protein from the fruits of Lycium ruthenicum. J Agric Food Chem 60: 9424-9429, 2012.

9. Wang JH, Chen XQ and Zhang WJ: Study on hypoglycemic function of polysaccharides from Lycium ruthenicum Murr. fruit and its mechanism. Food Sci 30: 244-248, 2009.

10. Guo S, Copps KD, Dong X, Park S, Cheng Z, Pocai A, Rossetti L, Sajan M, Farese RV and White MF: The Irs1 branch of the insulin signaling cascade plays a dominant role in hepatic nutrient homeostasis. Mol Cell Biol 29: 5070-5083, 2009.

11. Malaguarnera M, Di Rosa M, Nicoletti F and Malaguarnera L: Molecular mechanisms involved in NAFLD progression. J Mol Med (Berl) 87: 679-695, 2009.

12. Musso G, Gambino R and Cassader M: Recent insights into hepatic lipid metabolism in non-alcoholic fatty liver disease (NAFLD). Prog Lipid Res 48: 1-26, 2009. 
13. Bijland S, Mancini SJ and Salt IP: Role of AMP-activated protein kinase in adipose tissue metabolism and inflammation. Clin Sci 124: 491-507, 2013.

14. Cohen JC, Horton JD and Hobbs HH: Human fatty liver disease: Old questions and new insights. Science 332: 1519-1523, 2011.

15. Rahimi RS and Landaverde C: Nonalcoholic fatty liver disease and the metabolic syndrome: Clinical implications and treatment. Nutr Clin Pract 28: 40-51, 2013.

16. Sanyal AJ, Campbell-Sargent C, Mirshahi F, Rizzo WB, Contos MJ, Sterling RK, Luketic VA, Shiffman ML and Clore JN: Nonalcoholic steatohepatitis: Association of insulin resistance and mitochondrial abnormalities. Gastroenterology 120: 1183-1192, 2001.

17. Utzschneider KM and Kahn SE: Review: The role of insulin resistance in nonalcoholic fatty liver disease. J Clin Endocrinol Metab 91: 4753-4761, 2006.

18. Serviddio G, Bellanti F and Vendemiale G: Free radical biology for medicine: Learning from nonalcoholic fatty liver disease. Free Radic Biol Med 65: 952-968, 2013.

19. Shimano H, Yahagi N, Amemiya-Kudo M, Hasty AH, Osuga J, Tamura Y, Shionoiri F, Iizuka Y, Ohashi K, Harada K, Gotoda T, et al: Sterol regulatory element-binding protein-1 as a key transcription factor for nutritional induction of lipogenic enzyme genes. J Biol Chem 274: 35832-35839, 1999.

20. Matsumoto M, Ogawa W, Akimoto K, Inoue H, Miyake K, Furukawa K, Hayashi Y, Iguchi H, Matsuki Y, Hiramatsu R, Shimano $\mathrm{H}$, et al: PKClambda in liver mediates insulin-induced SREBP-1c expression and determines both hepatic lipid content and overall insulin sensitivity. J Clin Invest 112: 935-944, 2003.

21. Rakhshandehroo M, Knoch B, Müller M and Kersten S: Peroxisome proliferator-activated receptor alpha target genes. PPAR Res 2010: 612089, 2010.
22. Reddy JK and Rao MS: Lipid metabolism and liver inflammation. II. Fatty liver disease and fatty acid oxidation. Am J Physiol Gastrointest Liver Physiol 290: G852-G858, 2006.

23. Cao H, Maeda K, Gorgun CZ, Kim HJ, Park SY, Shulman GI, Kim JK and Hotamisligil GS: Regulation of metabolic responses by adipocyte/macrophage fatty acid-binding proteins in leptin-deficient mice. Diabetes 55: 1915-1922, 2006.

24. Ahmed MH and Byrne CD: Modulation of sterol regulatory element binding proteins (SREBPs) as potential treatments for non-alcoholic fatty liver disease (NAFLD). Drug Discov Today 12: 740-747, 2007.

25. Kohjima M, Higuchi N, Kato M, Kotoh K, Yoshimoto T, Fujino T, Yada M, Yada R, Harada N, Enjoji M, Takayanagi R and Nakamuta M: SREBP-1c, regulated by the insulin and AMPK signaling pathways, plays a role in nonalcoholic fatty liver disease. Int J Mol Med 21: 507-511, 2008.

26. Barroso E, Rodriguez-Calvo R, Serrano-Marco L, Astudillo AM, Balsinde J, Palomer X and Vasquez-Carrera M: The PPAR $\beta / \delta$ activator GW501516 prevents the down-regulation of AMPK caused by high-fat diet in liver and amplifies the PGC-1 $\alpha$-lipin 1-PPAR $\alpha$ pathway leading to increased fatty acid oxidation. Endocrinology 152: 1848-1859, 2011.

27. Viollet B, Athea Y, Mounier R, Guigas B, Zarrinpashneh E, Horman S, Lantier L, Hebrard S, Devin-Leclerc J, Beauloye C, Foretz M, et al: AMPK: Lessons from transgenic and knockout animals. Front Biosci (Landmark Ed) 14: 19-44, 2009.

28. Li Y, Xu S, Mihaylova MM, Zheng B, Hou X, Jiang B, Park O, Luo Z, Lefai E, Shyy JY, Gao B, et al: AMPK phosphorylates and inhibits SREBP activity to attenuate hepatic steatosis and atherosclerosis in diet-induced insulin-resistant mice. Cell Metab 13: 376-388, 2011 